\title{
AN IT-BASED FRAMEWORK FOR KNOWLEDGE MANAGEMENT IN NETWORKED ORGANISATIONS
}

\author{
Luiz Márcio Spinosa, Carlos Quandt \\ Pontifical Catholic University of Paraná \\ SKM - Strategic Knowledge Management Group \\ $R$. Imaculada Conceição, 1155, P. Velho \\ CEP. 80215901 - Curitiba - PR - BRAZIL
}

Tel. (02141) 332-0133 Fax. (02141)332-3323

(spinosa@tecpar.br)

\begin{abstract}
In order to increase the competitive advantage in the near future, which will be oriented by the Knowledge Economy, the enterprises should be aware about and make use of Information Technologies solutions well-adapted for knowledge management requirements. In fact, the future competitiveness of networked economies will be determined by their ability to make effective use of new methods and technologies that are able to deal with intangible assets, such as information and knowledge. The reason leading the enterprises to be concerned is to transform ideas into commercialised products, i.e. to recover the individual knowledge - the intellectual capital - and transform it into products and services that can be exploited by the organisation. Such assertion is specially emphasised in virtual enterprise context and provides several open questions guiding to sensible research efforts.

Keywords : Knowledge Management, Networked Organisation, Information Technology, Software Engineering
\end{abstract}

\section{INTRODUCTION}

Aiming at improve the productivity and flexibility of production, as well as balance the use of capital, personal, information, etc., managers have been formulated in the last years several organisational approaches, amongst which the networked organisations. They provide conditions for a fast and agile reaction to market requirements by forming sets of business related and Internet connected enterprises. Products and services are provided by a supply-chain typically having a main enterprise delivering essential components, leaving for its suppliers the provision of raw materials and sub-components.

The formation of networked organisations by themselves do not achieve the required competitiveness. The implantation and use of Information Technology (IT) infrastructures ascends in significance to support the enterprises interactions. Through IT-based solutions the operation of a networked organisation basically consists on assure the commercial (production and/or supply orders), technical (product specification) and managerial (orders monitoring, planning control, etc.) 
flows of information in order to accomplish the upright execution of enterprises' activities.

However, for the definition of a really competitive advantage, it is necessary the adoption of policies assuring the generation, the use, the maintenance and the dissemination of the intellectual capital of the network, that is, the adoption of IT for Knowledge Management in the networked organisations.

Knowledge Management methods are justified in networked organisations since the knowledge of the individuals - the intellectual capital - emerges from the network and are transformed into products and services that can be commercially explored. New businesses opportunities are created contributing to the innovative process and thus assuring the success of enterprises, particularly the Technologybased enterprises. Knowledge has been pointed out as one of the most important property of an organisation (Harris, 1999). It can improve the products' quality and services, accelerate the resolution of problems and better fit the market changes (Beckman, 1999) (Beckman, and Liebowitz, 1998). In fact, the acknowledgement of the expressions such as "New Economy" and "Knowledge-based Economy" results of the perception of the vital role of the knowledge and the technology in the economic growth (OCDE, 1996).

An adequate management of knowledge allows managers to get answers to some strategical questions, for instance: Which has been the predilection of my clients? Where are the bigger problems of quality? Where the clients are located? How have been the behaviour of the market related with importation and exportation flows? Is there a current business opportunity for my enterprise? Where my competitors are earning more?

The paper addresses those issues, initially providing a survey on knowledge management requirements for networked organisations. Afterwards, a general framework on IT is proposed to cope with those requirements. The framework is also quite related with virtual enterprise concerns, mainly those concerning the supply chain management issues. In fact, the success of virtual enterprises is guided by the need to improve the performance of supply chains through Information Technology in order to cope with strategical alliances and, at the same time, promote the Knowledge Management. Finally, some conclusions are provided.

\section{KNOWLEDGE MANAGEMENT REQUIREMENTS}

Sveiby (1999) identifies two basic approaches of knowledge management. The first one considers the knowledge management as an specific management of information, where knowledge is equivalent to objects that can be identified and treated by information systems. In the second one, knowledge management is equivalent to the management of people, and knowledge is equivalent to processes, as a dynamic set of qualifications and know-how leading to the learning and creation of abilities. Moreover, from the originally proposed classification of Polanyi (Liebowitz, 1999), it is possible to identify two basic types of knowledge in the organization: explicit (or codified) and tacit (Nonaka and Takeuchi, 1997). The explicit knowledge corresponds to procedures, databases, patents and relationships with customers, and can be codified, stored, and transmitted through formal way. It 
represents a small collection of all the knowledge body. The tacit knowledge is less formalized. It basically makes reference to experience, to the power of innovation and the ability of people. It is transmitted with more difficulty, mainly through examples (best-practices), and can originate new applications, depending on each context and the experience of each individual. When both tacit and implicit kinds of knowledge are captured and incorporated to the systems, processes, products, norms and the culture of an organization, it it possible to take great advantage of the organizacional knowledge to support its actions (Myers, 1996) (Stewart (1998).

The present work assumes the above classification from a more pragmatical perspective. The knowledge management claims to the explicit and systematic management of the vital knowledge and its associated processes (Liebowitz, 1999) (Murray and Myers. 1999). In this sense, the basic requirement on knowledge management, as far as IT-based solutions are considered, is take into account the general knowledge value chain (Figure 1). In fact, IT-based solutions have to support the creation, organisation, broadcasting, use, examination and protection of knowledge.

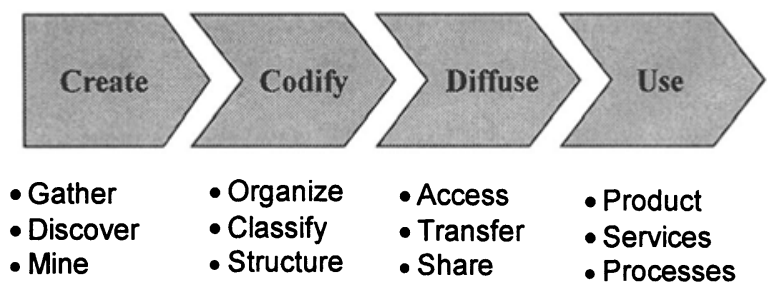

Figure 1 - Knowledge Value Chain

The objective is to connect problems to solutions in an efficient and fast manner. This implies in becoming the knowledge of individuals in corporate knowledge to be shared and duly applied by the organisation. In this sense, the nature of knowledge is concerned and the IT-based solutions for knowledge management have to prioritise:

- the knowledge of clients. No productive structure is currently conceived without take into account client's requirements, which represent one of the major determinant in the innovative process of enterprises. The clients' feedback is necessary during the design process of products and services, involving expectations of use, maintenance and recycling, as well as in the planning of the production as a whole. A "suitable information channel" must be opened to acquire those requirements;

- the knowledge of products. It is a fact that good knowledge about products can facilitate their commercialisation and, mainly, encourage innovative modifications leading to new products. Make available that knowledge to the network members becomes essential, particularly the sales units. Salesmen, which usually have direct contact with the clientele, can idealise new products generating innovative knowledge, which must be duly captured;

- the knowledge of processes. The quality of products and services, as well as the ability of enterprises in absorbing the market requirements can be attributed to 
the flexibility they have in customising their processes. The flexibility is only possible through the perfect understanding (knowledge) of the processes;

- the knowledge of relations. Usually, the establishment of strategical alliances in networked organisations requires great efforts to identify potentials partners in a truthful and fast form. Such requirements are quite important to rework existing relations, as well as to establish new agreements on sales and/or purchases among members. The required dynamic is only possible whether reliable knowledge about relationships can be provided;

- the knowledge of businesses. The uniform monitoring of the market behaviour can lead the enterprises to redirect their strategies in a faster and efficient form. That is the case of commodities, where quotations of products can have great weight in internal and external negotiation processes.

It shall be noted that behind all those natures the knowledge of people is the actual asset to be managed. In fact, the major challenge is : (i) to promote interactions among workers to come out their tacit knowledge; (ii) to convert the tacit knowledge into more explicit knowledge through documents, processes, databases, etc. and (iii) to spread the explicit knowledge into the networked organisation observing the right place, the right moment, the right people and the right content of information.

\section{GENERAL APPROACH}

The proposed platform takes into account the previously identified requirements and suggests that a set of IT-based services have to be installed on each enterprise composing the networked organization. See Figure 2. Those services can be organized on two logical levels:

- Functionalities, techniques and methods able to support the Life Cycle of networked organizations, typically characterized by the Formation, Operation, Coordination and Dissolution of the networks. The emphasis is on safe, fast and trustful transaction of technical and commercial information among the enterprises.

- Functionalities, techniques and methods able to support the Life Cycle of the Knowledge Management in networked organizations. Normally, such life cycle is summarized on four phases: Creation or Identification, Coding, Diffusing and Use. An additional set of functionalities consider the Protection of the Knowledge. 
Networked Enterprise
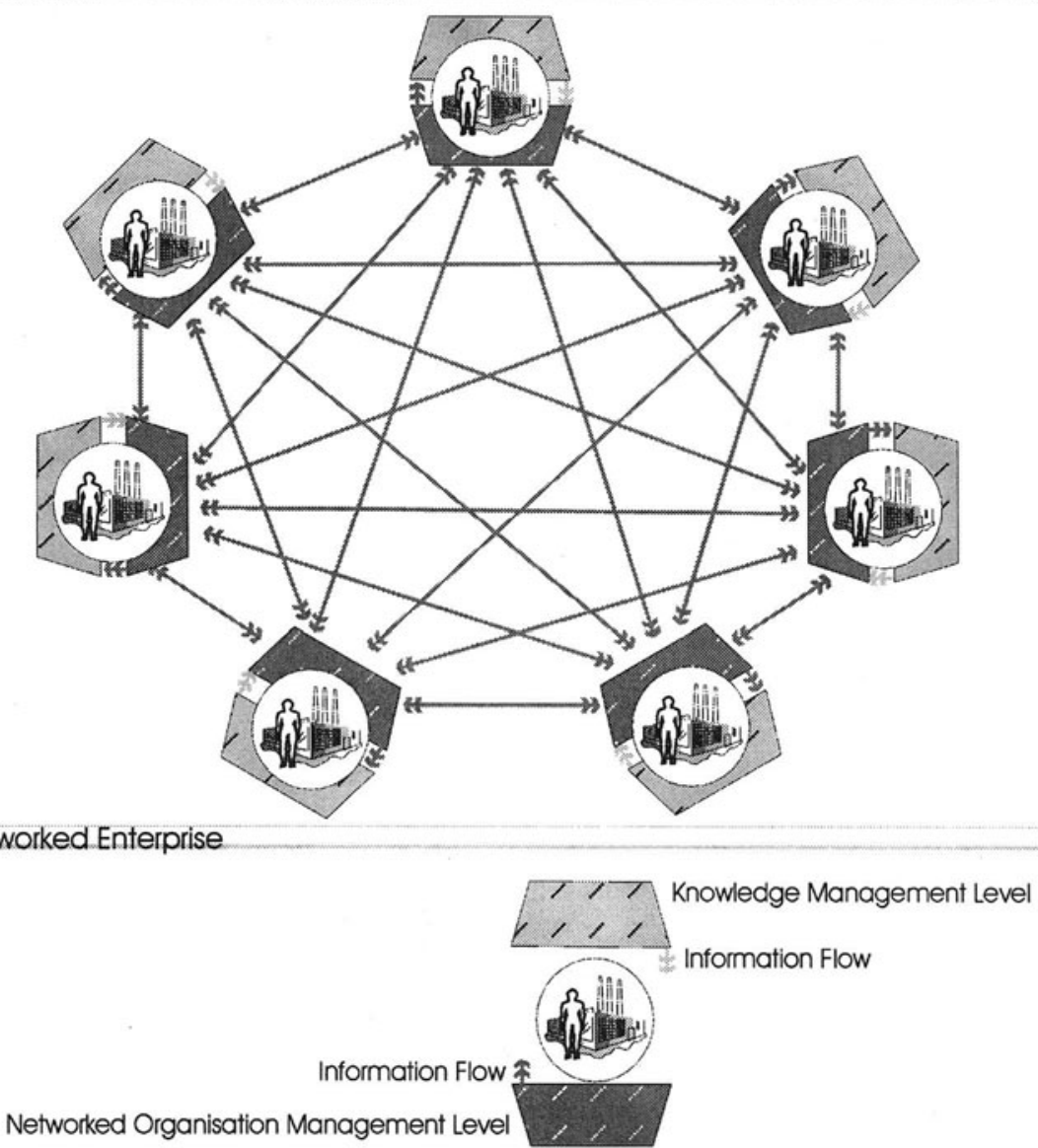

Figure 2-General Approach : The Networked Enterprises are extended with the ITbased Knowledge Management Framework.

One of the biggest challenges of this proposal is conceive and develop the services observing the integration of the two considered Life Cycles. Thus, the Modules were idealized in order to contemplate a reliable exchange of information among them.

\section{FUNCTIONALITIES FOR NETWORKED ORGANISATION MANAGEMENT}

A reasonable number of programs and projects have been devoted to the operation and co-ordination of networked organisations, particularly in United States, Europe and more recently in Brazil. Those projects suggest that major part of enterprises interactions are made by Internet, establishing a very important IT ground. A valuable case is the Prodnet-II Project (Camarinha et al, 1997), which depicts a set 
of services here taken as a generic representation of those programs and projects. That set corresponds to the first logical level of the General Approach, for instance (Figure 3): (i) connection with Legacy System; (ii) securized communication inside and outside the network; (iii) technological information handling (STEP features); (iii) orders handling (EDIFACT features); (iv) orders supervision (monitoring and control); (iv) management of distributed business process (monitoring, control and decision support); (v) user interaction handling, and particularly (vi) management of distributed information.

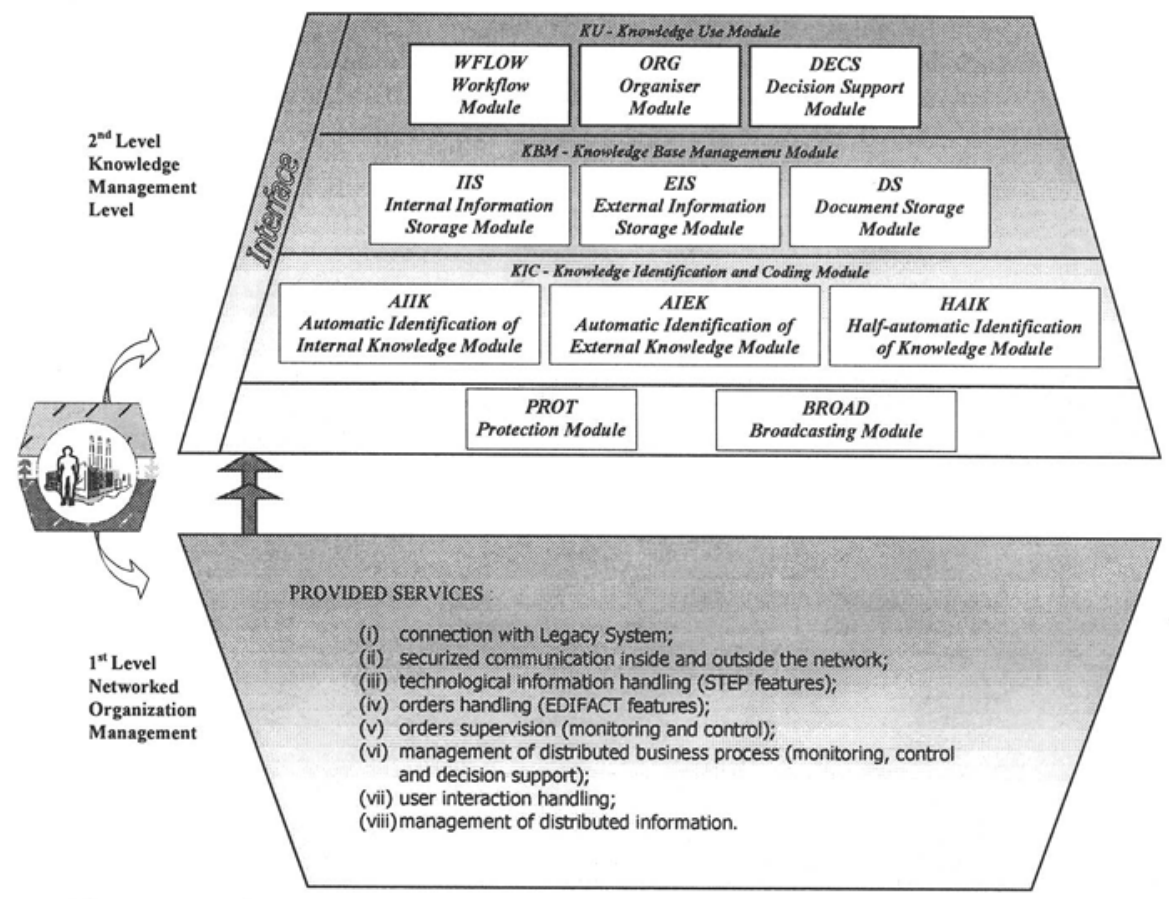

Figure 3 - The Knowledge Management Framework: services to manage the networked organisation (first level) and the networked organisation knowledge (second level).

Despite the importance of those services, they will not be detailed further considering the objective of this paper. In fact, the above functionalities can be considered as the basic set which support the definition of Knowledge Management Level following described.

\section{FUNCTIONALITIES FOR KNOWLEDGE MANAGEMENT}

The second logical level of the General Approach - Knowledge Management - uses the services to manage the networked organization (first level) and is able to fulfill most of the requirements for knowledge management in networked organizations. It is defined by the following services (Figure 3): 
KIC - Knowledge Identification and Coding Module - This module is composed of:

- AIIK - Automatic Identification of Internal Knowledge Module - The information transiting in the networked organization can be interpreted giving rise to important experience on commercial and cooperative transactions. Such experience constitutes a competitive advantage for the enterprise and have to be identified, retrieved and analyzed to favor decisions in a fast and proper way. This sub-module fulfils such needs in an automatic way (very lower "human" intervention) focusing only on the internal information exchanged through the networked organization, particularly that stored in the Networked Organisation Management Level. The involved techniques and methods evolve : an adaptation of typical Data Mining Systems, through the use of Artificial Intelligence, particularly the Intelligent Agents, and dedicated network protocols.

- AIEK - Automatic Identification of External Knowledge Module - There is a similar need as far as the external information is concerned, which typically are public economic indicators (quotations of markets, taxes of exportation and importation, banking taxes, numbers provided by competitors, etc.) or business opportunities. It shall be noted that external information is more difficult to be apprehended considered its diversity and dynamics (always in change of formats). AIEK provides similar services compared to previous module and further services to deal with digitized information available on Data Bases, CD-ROMs and specialized sites on the Internet. AIEK can be seen as a Pull Technology or a kind of workflow technique engendering features able to follow up the external sources, with predefined period and formats, in a flexible way.

- HAIK - Half-automatic Identification of Knowledge Module - Information of great commercial value emerges from informal meetings, casual colloquies, discussion lists (internet-based), and reflection of the employees, establishing fruitful sources of solutions based on the experience of the people. A survey of such information can be done through an awareness policy, which "rewards" the employees whose information becomes useful for the enterprises. HAIK provides a support enabling specific edition features to easily describe the problems and their solutions coming out from that policy. Those features allow the retrieving of information already available in the network, leaving to the user the task to fulfil the "links" between the problems and the solutions, characterising a half-automatic edition.

KBM - Knowledge Base Management Module - All the knowledge identified by the previous Modules is managed in a logical structure, the KBM module, which is composed of the following sub-modules:

- IIS - Internal Information Storage Module - IIS gathers all the internal information used by the networked organisation and considered of interest for the management of the knowledge. More precisely, the internal information is that handled by the Automatic Identification of Internal Knowledge previously defined. It shall be noted that the information is not literally stored in IIS, only the references (logical location of the pieces of information) are stored and managed. Such approach allows a more reliable information, once considered the Module that 
generate the information will be also responsible for its maintenance, as well as prevents possible redundancies. IIS can be seen as an extension of the services for distributed information management located at the Networked Organisation Management Level, thus, of distributed nature.

- EIS - External Information Storage Module - The present Module stores the external information automatically retrieved, i.e. handled by the Automatic Identification of External Knowledge. It differs of the previous one by effectively storing the information. Such approach is justified by the volume of information that, otherwise, could overload the communication means in the network. The techniques used by EIS are those conventionally offered by Distributed Data Base Systems.

- DS - Document Storage Module - This Module can be considered a typical repository of data and stores two groups of information: strategical documents and best-practices. The former concerns manuals of products, manuals of procedures, guides, etc. The later concerns the description of successful cases. DS can be seen as a set of services to support the previously defined modules, i.e. the previous Modules constantly make calls to DS's services.

KU - Knowledge Use Module - The knowledge identified and organised by the preceding Modules must be put available in order to encourage the essential interaction among the workers and, thus, promote the emerging of new knowledge. $\mathrm{KU}$ assure such needs thanks to the following sub-modules:

- WFLOW - Workflow Module - It was postulated that the management of the knowledge obeys a cycle of activities involving identification, coding, dissemination, use and protection of the knowledge. Much of the success of a knowledge management strategy is based on a standardised and systemised definition of those activities. WFLOW aims at assure the life cycle of knowledge in the networked enterprises, defining a plan to execute their activities. In short, the activities and sub-activities are listed, commanded and attributed to the responsible workers for their accomplishment in the networked enterprise. Technically, WFLOW is based on concepts of the Workflow Management Coalition, and can be presented as an electronic publisher allowing to elaborate a logical project of activities execution.

- ORG - Organiser - Without an organised structure of the information stored by the previous Modules, the users (networked enterprises) will locate the information in a limited form, leaving them to decide on weak bases. Normally, the information is organised on " families" such as "knowledge on products", "knowledge on services", "knowledge on markets", "knowledge on customers ", etc. ORG offers the possibility to correlate, classify and filter the information in accordance with those " families" besides allowing fast, efficient and safe access. The techniques used by ORG are those suggested by the Electronic Management of Documents, further adapted to distributed nature of the knowledge families imposed by the network context.

- DECS - Decision Support Module - The knowledge acquired and codified in the Knowledge Base is really useful when capable to answer strategical questions from the managemental board of networked enterprises. DECS can be seen as the gate of access to the knowledge stored in the Base of Knowledge, serving as intelligent 
assistant for eventual decisions. Technically, DECS is composed of some basic functions: (i) a publisher of Decisional Protocols which, in short, offers a series of basic routines able to compose "scripts" guiding the decisions over the knowledge (the protocols), (ii) an inference machine enabling automated reasoning over the Knowledge Base (in the Knowledge Base Management Module), and (iii) an userfriendly Interface enabling the use of the both functions. It shall be noted that the "protocols" are specific for each case, or either, their definition observes the specifications of each networked enterprise.

BROAD - Broadcasting Module - The identified and codified knowledge have to be spread out guaranteeing the right information will be in the right place at the right moment. BROAD aims at provide a set of features for automatic sending of messages and news bulletins. Still, BROAD allows the creation and management of discussion lists which will be in part fed by the messages above cited, as well as for the participants themselves. To define the logic of "who" receives "what", BROAD uses the features of Workflow Module.

PROT - Protection Module - Despite the Knowledge Economy is object of current study, its value is unquestionable. Knowledge is an important capital of the enterprise and, as such, it must be protect. It becomes necessary the protection of the Knowledge Base, as well as the communications engaged by the Modules. PROT provides such protection which also implies a strategical perspective. In fact, entrepreneurs consent to share the knowledge of their enterprises in a network only if guarantees of their know-how will be safe of the undesirable use (competition for instance). The challenge to PROT is balance strategies to hinder the robbery of knowledge and, at the same time, not to avoid it access to those that can contribute to its progress. Encryptation, authentication of users, associates to mapping techniques over the Knowledge Base, are basic strategies to being followed by PROT. PROT can also use the securized communication services provided by the first level of the Framework.

UI - User Interface - UI assures the exchange of information with the human operator allowing common and standardised interaction procedures, which are used by the described already functionalities.

\section{CONCLUSION}

The future competitiveness of networked economies will be determined by their ability to make effective use of new methods and technologies that are able to deal with intangible assets, such as information and knowledge. The reason leading the enterprises to be concerned with such assertion is to transform ideas into commercialised products, i.e. to recover the individual knowledge - the intellectual capital - and transform it into products and services that can be exploited by the organisation. In this sense, an IT-based framework was proposed to cope with knowledge management requirements for networked organisations. The proposed framework, which have to be installed in each enterprise composing the networked 
organization, is organized on services able to support two inter-related management life cycles: (i) Formation, Operation, Coordination and Dissolution of the networks and (ii) Creation or Identification, Coding, Diffusing and Use of knowledge. Both sets are conceived from an integrated perspective.

That IT-based Framework helps to understand those important aspects mainly those concerned with the services implementation. Despite that, the framework is only a software engineering tool. It shall be noted that the skilful use of such tool requires the execution of a Knowledge Management Plan, which introduces in networked enterprises the "culture" for management of their intellectual capital. The practice shows that this Plan is the main factor of success for communities engaged on the Knowledge Economy. The deep understanding of the cited Framework and Plan concerns our present research which is developed in the Strategic Knowledge Management Group in the scope of the Master Degree on Strategical Use of Information at Pontifical Catholic University of Paraná.

\section{REFERENCES}

1. Beckman, T. 1999. The Current State of Knowledge Management. In: Liebowitz, J. (ed.) Knowledge Management Handbook. Mew York: CRC Press.

2. Beckman, T. e Liebowitz, J. 1998. Knowledge Organizations: what every manager should know. St. Luice Publications.

4. Harris, K. 1999. Enterprise IQ: The Knowledge-Enabled Firm. Commentary Document \#COM-091449, 27 August 1999, The Gartner Group.

5. Liebowitz, J. (ed.). 1999. Knowledge Management Handbook. Mew York: CRC Press.

6. Murray, P. e A. Myers. 1999. The Facts About Knowledge. Knowledge Management Survey, Cransfield School of Management. http://www.infostrategy.com/knowsur1/

7. Myers, P. (ed.) 1996. Knowledge Management and Organizational Learning. London: ButterworthHeinemann.

8. OCDE. 1996. The Knowledge Based Economy. Excerpted from the 1996 Science, Technology and Industry Outlook. Paris: OCDE.

9. Sveiby, K. 1999. What is knowledge management? http://www.sveiby.com.au/Knowledge Management.html

11. Nonaka, I. e H. Takeuchi. 1997. Criação de Conhecimento na Empresa. Rio de Janeiro: Campus.

12. Stewart, T. 1998. Capital Intelectual: A nova vantagem competitiva das empresas. Rio de Janeiro: Campus.

13. Camarinha-Matos, L., Lima, C., Osorio, A. L., The PRODNET platform for production planning and management in virtual enterprises, Proc. Of the $4^{\text {th }}$ International Conference on Concurrent Enterprising, 8-10 October 1997, Unversity of Nottinghan, UK. 